\title{
The Supraclavicular Artery Flap for Lateral Skull and Scalp Defects: Effective and Efficient Alternative to Free Tissue Transfer
}

\author{
Jason P. Hunt ${ }^{1}$ Luke O. Buchmann ${ }^{1}$ \\ ${ }^{1}$ Division of Otolaryngology, Department of Surgery, University of \\ Utah, Salt Lake City, Utah, United States \\ J Neurol Surg Rep 2014;75:e5-e10.
}

\begin{abstract}
Address for correspondence Jason P. Hunt, MD, Division of Otolaryngology, Department of Surgery, University of Utah, $50 \mathrm{~N}$. Medical Drive, 3C120 SOM, Salt Lake City, UT 84132, United States (e-mail: jason.hunt@hsc.utah.edu).
\end{abstract}

\begin{abstract}
Keywords

- supraclavicular flap

- lateral skull base

- reconstruction

- regional flap

Objectives Describe the use of the supraclavicular artery flap for reconstruction of lateral skull and scalp defects. Discuss advantages and potential limitations of the supraclavicular artery flap.

Design Case series.

Setting Tertiary care academic medical center.

Participants Patients undergoing lateral scalp and skull base resections.

Main Outcome Measures Effectiveness in reconstructing lateral skull base defects and complications.

Results All three patients reconstructed with the supraclavicular artery flap had excellent reconstructive outcomes. There were no flap losses, either complete or partial. There were no major complications, but one patient had a significant donor site dehiscence requiring local wound care. Referred sensation to the shoulder was alleviated by division of the sensory innervations into the flap.

Conclusions The supraclavicular artery flap is an excellent option for lateral skull and scalp defects, and donor site morbidity is limited. It should be considered as an alternative to free tissue transfer.
\end{abstract}

\section{Introduction}

Lateral scalp and auriculectomy defects can be a challenge for the reconstructive surgeon. They are often associated with lateral temporal bone and parotidectomy defects. These defects frequently arise in elderly patients with skin cancers and significant comorbid conditions in which limited operative time is preferred. Many of these defects are not amenable to local advancement flaps, and the surgeon must consider regional and distant options. An ideal reconstruction would provide a pliable versatile flap that could replace the skin with a good color match and be able to cover both thin and deep defects seen in lateral temporal bone resections. These options have included the temporalis muscle flap with skin grafts, lower island trapezius flaps, pectoralis major myocu- taneous flaps, among others. ${ }^{1-3}$ However, these options are not always ideal due to the long reach of these rotational flaps and frequent preoperative radiation. These factors make wound dehiscence and partial flap loss common. These limitations have led reconstructive surgeons to frequently use free tissue transfer for these defects, leading to increased surgical time and complexity. ${ }^{4}$

The supraclavicular artery flap (SCAF) was first described as an axial patterned flap by Lamberty in $1979 .{ }^{5}$ This flap was subsequently popularized in head and neck reconstruction by others including Pallua and Magnus Noah and Chiu et al. ${ }^{6,7}$ The SCAF has been described for multiple sites of reconstruction including oral cavity, trachea, pharynx, and skin defects. ${ }^{6-8}$ Advantages of the flap are the ease of harvest, minimal donor site morbidity, and lack of microvascular anastomosis. received

May 11, 2012

accepted after revision

June 27, 2013

published online

April 17, 2014
DOI http://dx.doi.org/

10.1055/s-0033-1358376. ISSN 2193-6358. (c) 2014 Georg Thieme Verlag KG
Stuttgart · New York

License terms

(1) $\circledast \circledast$ 
This report describes three cases of lateral scalp and auriculectomy defects reconstructed with the SCAF. We describe each case and discuss complications.

\section{Case Series}

\section{Flap Elevation}

All SCAFs were elevated in a similar fashion. The supraclavicular artery was identified with a handheld Doppler extending over the lateral clavicle approximately half the distance between the posterior border of the clavicular insertion of the sternocleidomastoid muscle and the anterior border of the trapezius muscle. The width of the SCAF was centered over the supraclavicular artery and designed over the deltoid as necessary to extend to the defect. The design can be seen in - Fig. 1 of the associated case. It was raised from distal to proximal in a subfascial plane over the deltoid musculature. At the clavicle, the periosteum was elevated and incised so as not to disrupt the supraclavicular artery. The vascular pedicle was identified in the fatty tissue in the supraclavicular fossa. The surrounding tissue was freed, and the distal branches of the transverse cervical vascular pedicle were ligated as necessary to allow tension-free rotation of the flap to the surgical defect. The flap was deepithelialized as necessary for tunneling under skin bridges to reach the defect and inset. The donor site was closed by elevating surrounding tissue in a subcutaneous plane to allow advancement and closure.

\section{Case One}

A 78-year-old man with a history of chronic lymphocytic leukemia presented to the Head and Neck Oncology Clinic with a history of a multiply recurrent pleomorphic adenoma of the parotid treated with multiple surgeries and radiation

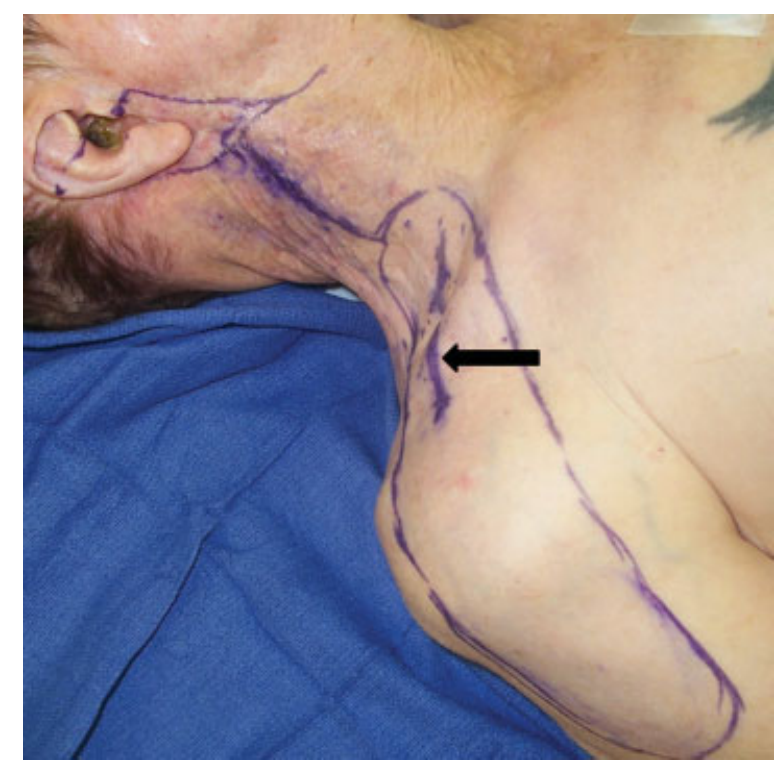

Fig. 1 Surgical plan. The supraclavicular artery flap (SCAF) design is shown with proximal aspect of the supraclavicular artery outlined running over the lateral aspect of the clavicle (arrow).

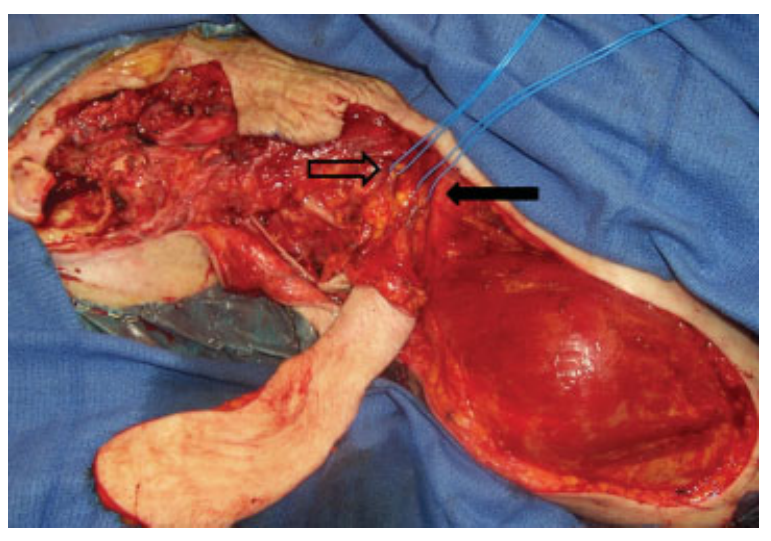

Fig. 2 Intraoperative photograph. The lateral temporal bone defect is seen with the supraclavicular artery flap elevated. The pedicle of the flap (dark arrow) and the sensory nerve (open arrow) innervating the flap can be seen.

therapy. On presentation to the head and neck clinic, the tumor was noted to have invaded the external auditory canal. Tumor resection required partial auriculectomy, radical parotidectomy, and lateral temporal bone resection leaving a defect measuring $6 \times 6 \mathrm{~cm}$, and the final diagnosis proved to be a carcinoma expleomorphic adenoma. -Fig. 1 shows the planned excision and SCAF design. Reconstruction included a medial antebrachial cutaneous nerve graft for the facial nerve and a pedicled SCAF for defect closure. -Fig. 2 shows the defect with the flap elevated. Of note, a cervical rootlet was ligated because it was seen traversing from the midportion of the posterior border of the sternocleidomastoid to the flap. This flap was inset into the neck incision as shown in the immediate postoperative period in - Fig. 3. Early in our use of the SCAF, limited deepithelialization was performed. The postoperative course was uncomplicated, and the patient was discharged on postoperative day 3 without complications. At the 2-week postoperative visit, the patient was doing well without complications and no decrease in strength or range of motion of the shoulder at the donor site. At the 3-month postoperative visit, the patient had no complaints, and no referred shoulder sensation was noted along the flap.

\section{Case Two}

An 84-year-old man presented to the Head and Neck Oncology Clinic with a recurrent squamous cell carcinoma of the

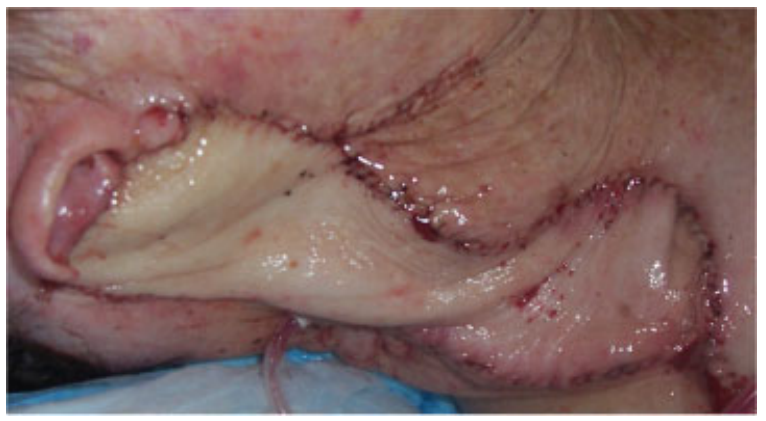

Fig. 3 Immediate postoperative result. The flap can be seen incorporated into the neck incision. 


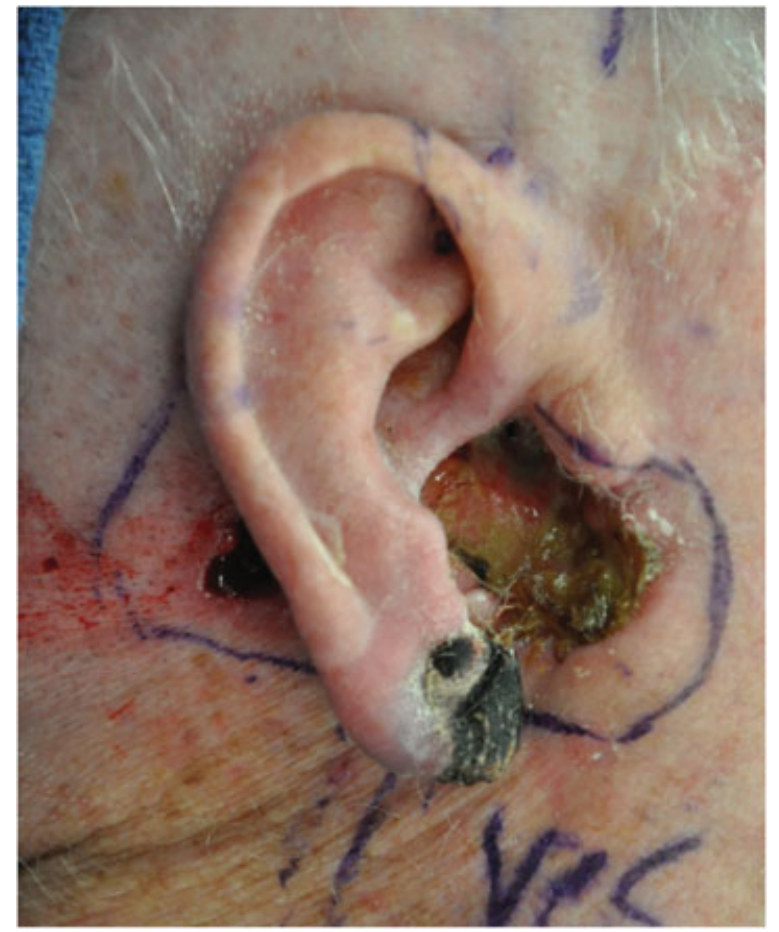

Fig. 4 Preoperative photograph of malignancy. The ulcerative lesion with biopsy-proven recurrent squamous cell carcinoma is seen involving a large portion of the auricle and ear canal.

right auricle with an associated ulcer (-Fig. 4). He had undergone multiple previous surgeries as well as radiation therapy prior to this recurrence. His associated comorbidities included noninsulin-dependent diabetes mellitus. After preoperative counseling, the patient was scheduled for an auriculectomy with lateral temporal bone resection as well as parotidectomy and neck dissection. Reconstructive options of a regional SCAF versus a free tissue transfer were discussed with the patient; however, it was decided that a regional reconstruction would be best in this patient because of his advanced age. Due to prior radiation and the expected size of

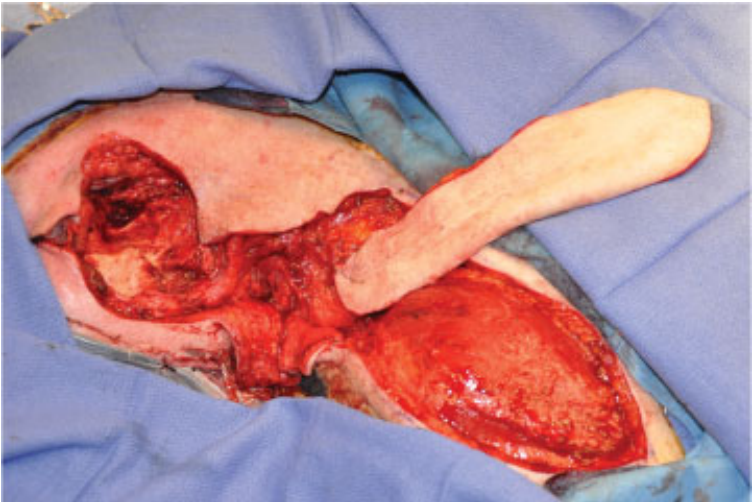

Fig. 5 Intraoperative photograph of defect and supraclavicular artery flap (SCAF). The open defect from auriculectomy and lateral temporal bone resection is seen along with the SCAF elevated prior to inset.

the defect, a temporalis muscle flap was not believed to be a good option.

The patient underwent resection as planned producing a $6 \times 7 \mathrm{~cm}$ defect with a significant deep dimension related to the temporal bone resection. A SCAF was elevated as shown along with the defect in - Fig. 5. The cervical rootlets were not isolated and ligated as they entered the flap over the clavicle. The proximal portion was deepithelialized and tunneled under the neck flap. To better reconstruct the depth of the wound, the distal portion of the flap was also deepithelialized and folded in to the temporal bone defect. The patient was discharged on postoperative day 3 . The patient healed well without complication as shown at his 1-month postoperative visit (-Fig. 6). At 3 months postoperatively, the patient was doing well without decrease in strength or range of motion of the donor site shoulder. His only complaint was referred sensation to the shoulder from the flap.

\section{Case Three}

A 41-year-old man with a history of an enlarging mass on his scalp posterior to the right auricle presented to the Head and

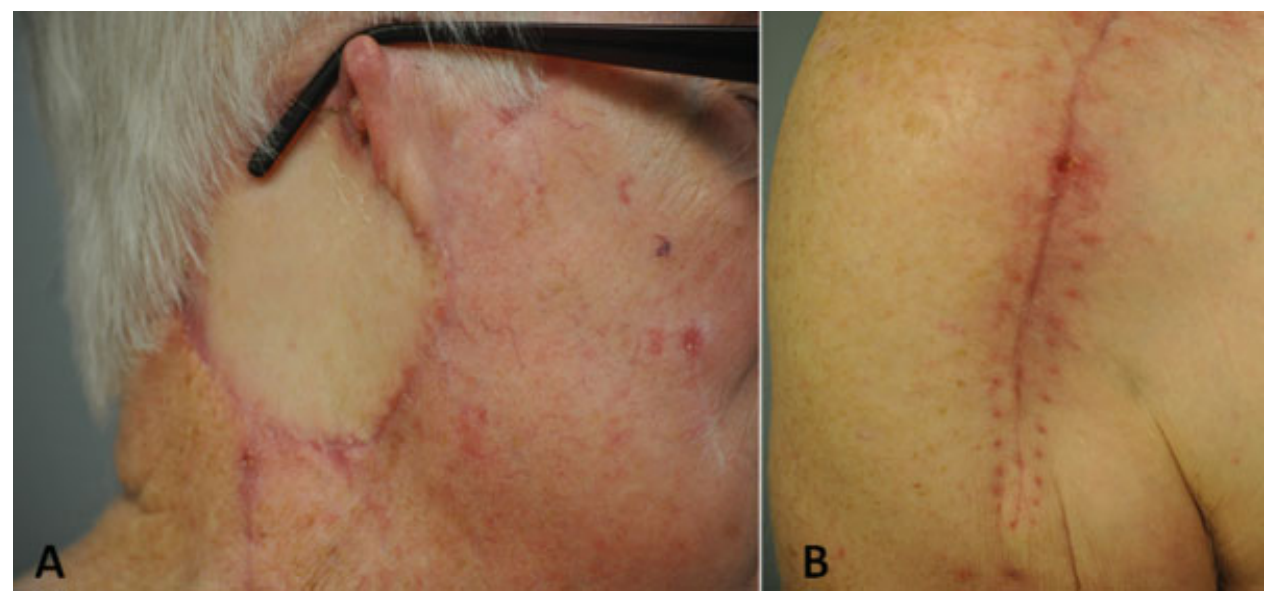

Fig. 6 Postoperative photograph at 1 month. (A) The wound can be seen well healed with an excellent contour. (B) The donor site is intact with mild widening of the incision. 


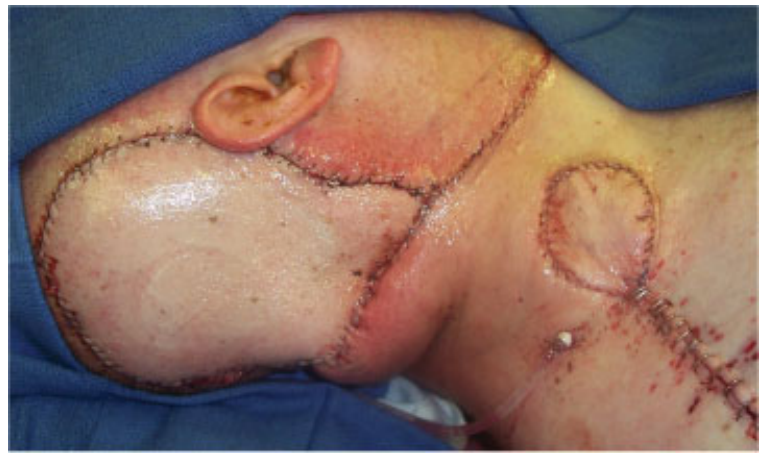

Fig. 7 Immediate postoperative photograph. The supraclavicular artery flap (SCAF) provides excellent coverage of the $10 \times 12 \mathrm{~cm}$ defect of the posterolateral scalp and is able to reach $\sim 4 \mathrm{~cm}$ above the auricle. To assist with closure of the donor defect, a proximal portion of the SCAF was incorporated into the defect. The intervening flap was deepithelialized and tunneled under the skin flap.

Neck Surgical Oncology Clinic. He was otherwise healthy. A biopsy of the lesion showed a leiomyosarcoma. He was scheduled for surgical resection and reconstruction. Regional flap reconstructive options and free tissue transfer options were discussed with the patient. The patient underwent a wide local excision, partial craniectomy, and selective neck dissection leaving a defect that measured $10 \times 12 \mathrm{~cm}$. An ipsilateral SCAF was harvested for reconstruction and tunneled under the neck skin to close the defect ( - Fig. 7). The cervical rootlet seen entering the flap territory was isolated and ligated at the posterior aspect of the sternocleidomastoid. A small portion of the flap was used to close the upper aspect of the donor site with the intervening flap tunneled under the inferior neck flap. The remaining donor site defect was closed via undermining and performing an adjacent tissue transfer. Postoperatively, the patient was admitted to the inpatient unit and discharged on postoperative day 4 . At 2 weeks postoperatively, staples were removed from the arm and a dehiscence of $2 \times 3 \mathrm{~cm}$ occurred. Local wound care was provided and the donor site wound closed by secondary intention in 12 weeks without long-term sequela (-Fig. 8). The scalp reconstruction remained durable during radiation (-Fig. 9). There was no evidence of referred sensation from the flap to the shoulder. The patient did have some decreased range of motion in the ipsilateral shoulder. This was believed to be a result of an 11th nerve paresis and recovered by the 12-week postoperative visit.

\section{Discussion}

The supraclavicular artery flap has made a resurgence in the reconstruction of the head and neck over the past decade. It consists of thin pliable tissue that is easily harvested in the supine position in $<1$ hour. $^{7}$ The donor site morbidity is minimal and well tolerated by patients. This series demonstrates the expanding versatility of the SCAF, and it provides an excellent reconstructive option for auriculectomy and lateral scalp defects with or without temporal bone resection. Pointer et al reported a similar reconstruction with a good result in a single case. ${ }^{9}$ The current studies shows continued excellent results in a series of three patients with a larger variety of wounds. Also, one patient in the current series required folding of the flap to fill in the temporal bone resection for appropriate depth. This technique adds versatility to this flap, allowing increased thickness to the flap for deeper defects. In the current series, all patients tolerated the procedure well. There were no recipient site complications,

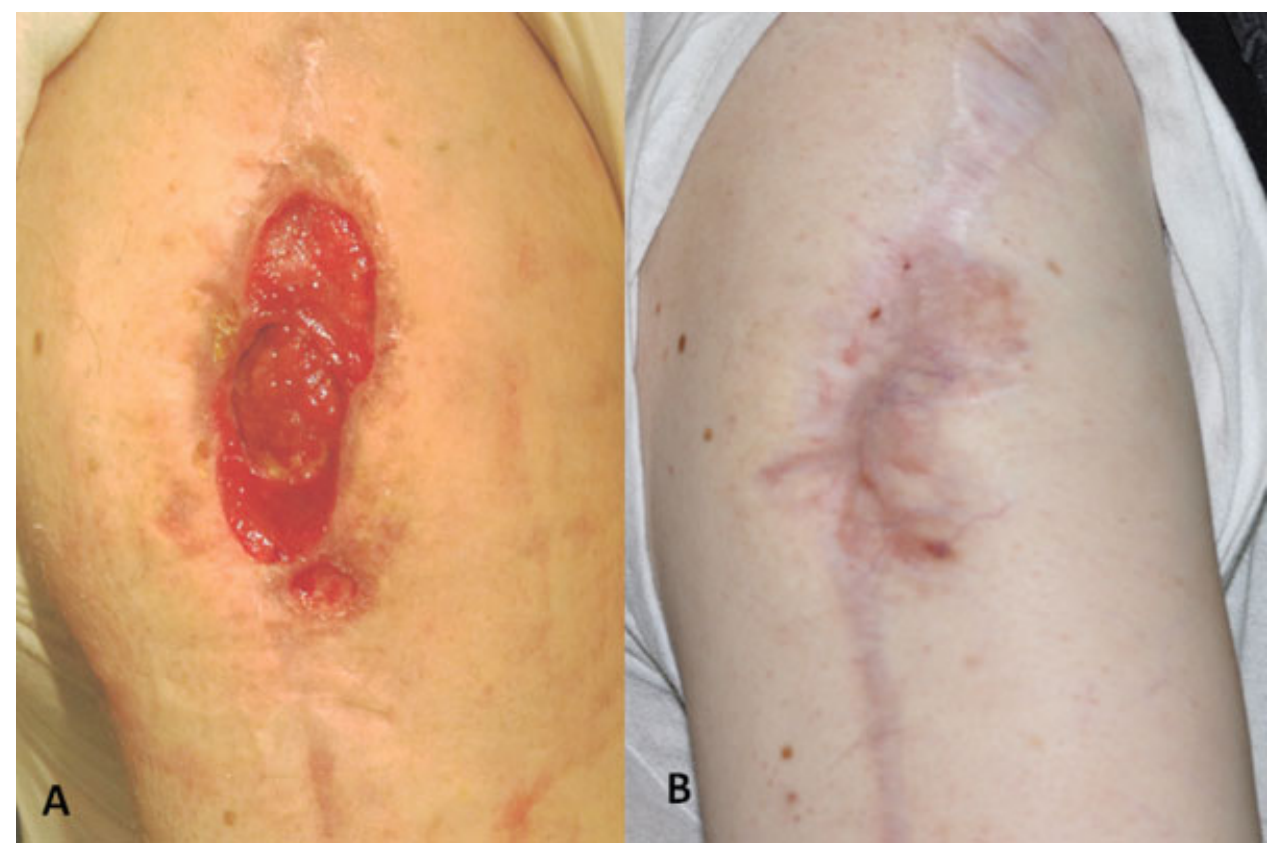

Fig. 8 Donor site wound dehiscence. (A) The donor site is shown with dehiscence of the midportion of the donor site incision. (B)The donor site is completely closed by 12 weeks without long-term sequela. 


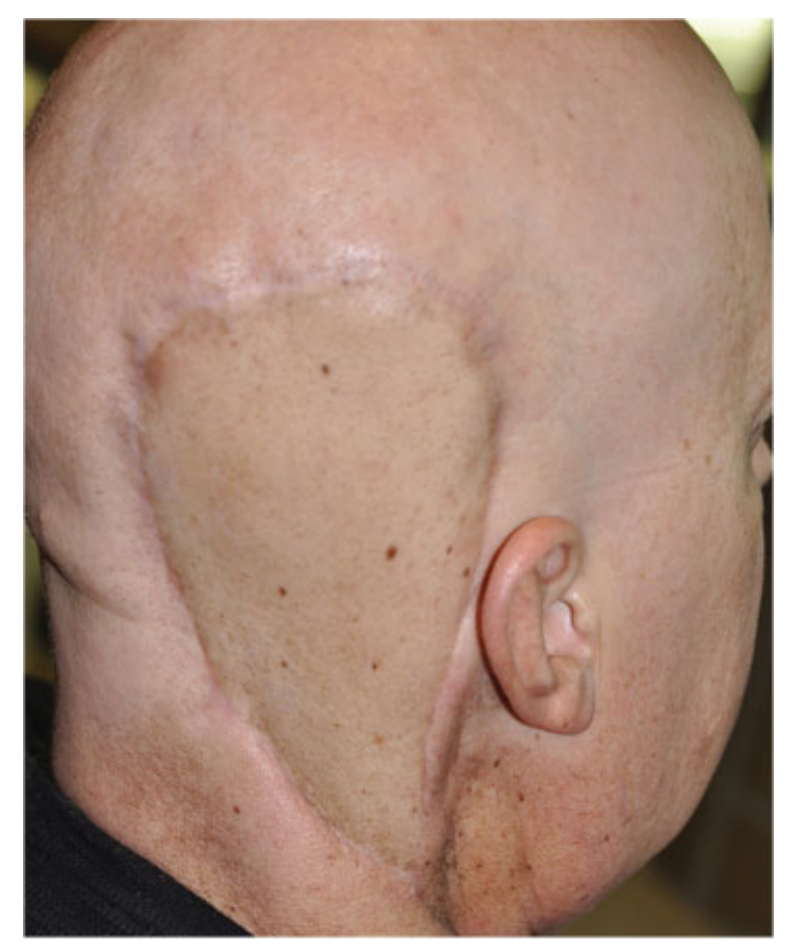

Fig. 9 Postoperative photograph after postoperative radiation. The supraclavicular artery flap is shown without complication and good contour with the rest of the scalp.

and all reconstructions were deemed acceptable by surgeon and patient. There was one minor donor site complication when a large defect was reconstructed with a 10-cm-wide flap. The donor site was closed with an adjacent tissue transfer under slight tension. The wound dehiscence occurred on postoperative day 12 after removal of staples and required several weeks of conservative therapy for closure. Donor site complications have been reported in up to18\% of cases in small series including wound dehiscence. ${ }^{10}$ Flap width of 9 to $10 \mathrm{~cm}$ is likely the limit of flap while allowing the donor site to be closed without skin grafts. Chiu et al suggested considering skin graft coverage for flaps $>8 \mathrm{~cm} .^{7}$ Others have reported the use of skin grafts when the width is $>10 \mathrm{~cm} .{ }^{11}$

One minor complaint regarding the SCAF is the referred sensation from the flap to the shoulder region. This was described in the past and related to the flap being transferred with sensory neural innervation intact. ${ }^{7}$ Although this is normally a minor nuisance, some patients may be troubled by it. The pattern of neural innervation has been described from cadaveric studies with branches from the cervical rootlets $(\mathrm{C} 3, \mathrm{C} 4)$ entering the flap separately from the vascular pedicle. $^{12}$ In the current series, this was noted as well. Patients with division of these cervical branches experience no referred pain. This sensation may be advantageous in some applications of pharyngeal and oral cavity reconstruction, but it is more of an annoyance in skin reconstruction, and we recommend division of these branches for similar types of reconstruction.

With such limited complications and excellent outcomes in this small series, we have observed that the SCAF is an excellent choice for lateral scalp defects including lateral temporal bone defects. Outside of the SCAF, other options have significant drawbacks. Local flaps of temporalis muscle with skin grafts are appropriate for small defects but are poor options for large defects and frequently have complications in a radiated field. Furthermore, temporalis muscle flaps are associated with a significant rate of trismus and leave a significant donor site defect unless an implant is used. ${ }^{13}$ Limitations on reconstruction include inadequate bulk to reconstruct deep defects of temporal bone resections. More distant regional flaps have greater utility for previously radiated patients including trapezius and pectoralis flaps. All of these options are limited by their significant bulk and limited length with the potential for partial flap loss. Although the lower island trapezius flap is a better match with appropriate length in most cases, like the latissimus flap, it requires the patient to be in the lateral decubitus position. For these reasons, many reconstructive surgeons have turned to free tissue transfer from various sites including latissimus dorsi, radial forearm, rectus, and anterolateral thigh, among others. ${ }^{4,14}$ These have a variety of potential donor site complications but provide an excellent reconstruction for a variety of defects. However, they do increase the complexity of the surgery with microvascular anastomoses required and extensive monitoring and hospital stay. For older patients with comorbidities, limited surgical time is desired, making free tissue transfer less attractive. Hospital stay is also likely to be less in patients reconstructed with a regional flap. Hospital stay in most patients with free tissue transfers is at a minimum 5 days with 2 to 3 days in high-cost units for flap monitoring. In a study by Hanasono et al, the average hospital stay was 9.6 days with an average of 2.8 days in an intensive care unit. ${ }^{14}$ This substantial cost of care would be much higher than the 3 to 4 days seen in the current study with no intensive care unit or intermediate care unit stays. Admittedly, there are patients with complicated wounds that will still require free tissue transfer including massive defects with significant depth. Also, defects extending significantly above the auricle and those with large dural defects should consider free tissue transfer. In case 3 of the current study, the defect extended over $4 \mathrm{~cm}$ above the auricle with good closure with the SCAF and no distal tip necrosis, demonstrating the excellent reach of the flap.

Some limitations of the SCAF warrant mentioning. It should be approached with caution in patients with previous ipsilateral neck dissections in which the transverse cervical artery may have been compromised because the supraclavicular artery is a branch of the transverse cervical artery in most cases. This can be evaluated with computed tomography with angiography in cases concerned for loss of the pedicle such as previous neck dissection. ${ }^{15}$ Also, flaps wider than $\sim$ $10 \mathrm{~cm}$ are unlikely to be closed without grafts. ${ }^{11}$ Flaps $<10 \mathrm{~cm}$ in width can be closed with significant undermining in two directions, but they frequently leave a widened scar. Otherwise, there is referred sensation to the shoulder, but anatomical studies suggest the neural innervations can easily be identified and ligated. ${ }^{16}$ This was performed in two cases in the current series with excellent results. There may 
be cases in which sensation would be useful in the pharynx or oral cavity, but sensory retraining may be required.

\section{Conclusion}

The SCAF is a versatile flap for reconstruction of head and neck defects and an excellent option in lateral skull and/or auriculectomy defects. The cosmetic results are excellent, and patient morbidity is limited. It should especially be considered in patients with comorbidities that warrant limiting surgical time but likely gives equivalent results to free tissue transfer with improved color match.

\section{References}

1 Gal TJ, Kerschner JE, Futran ND, et al. Reconstruction after temporal bone resection. Laryngoscope 1998;108(4, Pt 1):476-481

2 Netterville JL, Wood DE. The lower trapezius flap. Vascular anatomy and surgical technique. Arch Otolaryngol Head Neck Surg 1991;117(1):73-76

3 Resto VA, McKenna MJ, Deschler DG. Pectoralis major flap in composite lateral skull base defect reconstruction. Arch Otolaryngol Head Neck Surg 2007;133(5):490-494

4 O'Connell DA, Teng MS, Mendez E, Futran ND. Microvascular free tissue transfer in the reconstruction of scalp and lateral temporal bone defects. J Craniofac Surg 2011;22(3):801-804

5 Lamberty BG. The supra-clavicular axial patterned flap. Br J Plast Surg 1979;32(3):207-212

6 Pallua N, Magnus Noah E. The tunneled supraclavicular island flap: an optimized technique for head and neck reconstruction. Plast Reconstr Surg 2000;105(3):842-851; discussion 852-854
7 Chiu ES, Liu PH, Friedlander PL. Supraclavicular artery island flap for head and neck oncologic reconstruction: indications, complications, and outcomes. Plast Reconstr Surg 2009;124(1):115-123

8 Pallua N, Wolter TP. Defect classification and reconstruction algorithm for patients with tracheostomy using the tunneled supraclavicular artery island flap. Langenbecks Arch Surg 2010;395(8): 1115-1119

9 Pointer DT Jr, Friedlander PL, Amedee RG, Liu PH, Chiu ES. Infratemporal fossa reconstruction following total auriculectomy: an alternative flap option. J Plast Reconstr Aesthet Surg 2010; 63(8):e615-e618

10 Nthumba PM. The supraclavicular artery flap: a versatile flap for neck and orofacial reconstruction. J Oral Maxillofac Surg 2012; 70(8):1997-2004

11 Vinh VQ, Van Anh T, Ogawa R, Hyakusoku H. Anatomical and clinical studies of the supraclavicular flap: analysis of 103 flaps used to reconstruct neck scar contractures. Plast Reconstr Surg 2009;123(5):1471-1480

12 Sands TT, Martin JB, Simms E, Henderson MM, Friedlander PL, Chiu ES. Supraclavicular artery island flap innervation: anatomical studies and clinical implications. J Plast Reconstr Aesthet Surg 2012;65(1):68-71

13 Eldaly A, Magdy EA, Nour YA, Gaafar AH. Temporalis myofascial flap for primary cranial base reconstruction after tumor resection. Skull Base 2008;18(4):253-263

14 Hanasono MM, Sacks JM, Goel N, Ayad M, Skoracki RJ. The anterolateral thigh free flap for skull base reconstruction. Otolaryngol Head Neck Surg 2009;140(6):855-860

15 Adams AS, Wright MJ, Johnston S, et al. The use of multislice CT angiography preoperative study for supraclavicular artery island flap harvesting. Ann Plast Surg 2012;69(3):312-315

16 Sands TT, Martin JB, Simms E, Henderson MM, Friedlander PL, Chiu ES. Supraclavicular artery island flap innervation: anatomical studies and clinical implications. J Plast Reconstr Aesthet Surg 2012;65(1):68-71 